\title{
Real-time correction of respiratory-induced cardiac motion during electroanatomical mapping procedures
}

\author{
R. van $\mathrm{Es}^{1} \cdot$ F. J. van Slochteren ${ }^{1,2} \cdot$ S. J. Jansen of Lorkeers ${ }^{1} \cdot$ R. Blankena ${ }^{1,3}$. \\ P. A. Doevendans ${ }^{1} \cdot$ S. A. J. Chamuleau ${ }^{1}$
}

Received: 13 February 2015 / Accepted: 29 January 2016 / Published online: 25 March 2016

(c) The Author(s) 2016. This article is published with open access at Springerlink.com

\begin{abstract}
Treatment planning during catheter interventions in the heart is often based on electromechanical tissue characteristics obtained by endocardial surface mapping (ESM). Since studies have shown respiratory-induced cardiac motion of over $5 \mathrm{~mm}$ in different directions, respiratory motion may cause ESMs artifacts due to faulty interpolation. Hence, we designed and tested a real-time respiration-correction algorithm for ESM. An experimental phantom was used to design the correction algorithm which was subsequently evaluated in five pigs. A piezorespiratory belt transducer was used to measure the respiration. The respiratory signal was inserted to the $\mathrm{NOGA}^{\circledR} \mathrm{XP}$ electromechanical mapping system via the ECG leads. The results of the correction were assessed by measuring the displacement of a reference point and the registration error of the ESM on a CMR scan before and after correction. In the phantom experiment, the reference point displacement was $6.5 \mathrm{~mm}$ before and $1.1 \mathrm{~mm}$ after correction and the registration errors were $2.8 \pm 2.2$ and $1.9 \pm 1.3 \mathrm{~mm}$, respectively. In the animals, the average reference point displacement (apex) was reduced from $2.6 \pm 1.0 \mathrm{~mm}$ before to $1.2 \pm 0.3 \mathrm{~mm}$ after correction $(P<0.05)$. The in vivo registration error of the ESM and the CMR scan did not significantly improve. Even though the apical movement
\end{abstract}

R. van Es

R.vanes-2@umcutrecht.nl

1 Department of Cardiology, University Medical Centre Utrecht, E03.511, P.O. Box 85500, 3508 Utrecht, The Netherlands

2 Interuniversity Cardiology Institute of the Netherlands (ICIN), Utrecht, The Netherlands

3 Department of Technical Medicine, Faculty of Science and Technology, University of Twente, Enschede, The Netherlands appreciated in pigs is small, the correction algorithm shows a decrease in displacement after correction. Application of this algorithm omits the use of the time-consuming respiratory gating during ESM and may lead to less respiratory artifacts in clinical endocardial mapping procedures.

Keywords Electroanatomical mapping .

Respiratory-induced cardiac motion $\cdot$ CMR $\cdot$ Cardiac imaging

\section{Introduction}

Treatment planning during catheter interventions in the heart is often based on electromechanical tissue characteristics $[2,4]$ obtained by endocardial surface mapping (ESM). ESM systems determine the exact, real-time 3-dimensional (3D) location of the catheter tip inside the heart using an electrical or magnetic field, induced in the thorax of the patient $[2,16]$. Measurements of the local depolarization potentials and relative cardiac wall motion are taken on the endocardium and stored with their respective 3D locations to create a 3D reconstruction of the endocardium. Data are interpolated in the areas that are spatially located between the acquired mapping points to reconstruct a continuous endocardial surface.

During the ESM procedure, the patient is usually awake and breathing freely. Respiratory-induced cardiac motion (RICM) leads to spatial errors in the ESM. A low spatial resolution of ESM together with the RICM-induced spatial artifacts may cause inaccurate positioning of endocardial ablations or intramyocardial injections in the context of cardiac regenerative therapy. Studies in patients using different cardiac imaging modalities report RICM ranging up to a centimeter during free breathing, mainly in the 


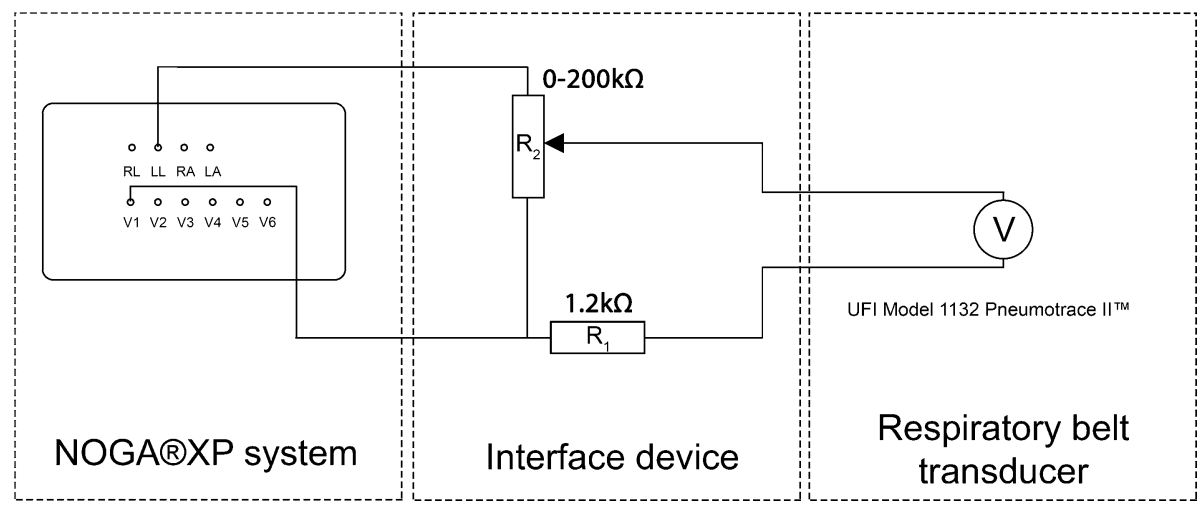

Fig. 1 Circuit of the system used to connect a respiratory belt transducer to the ECG leads of NOGA ${ }^{\circledR} \mathrm{XP}$ system to instigate ECG baseline drift in the V1 ECG channel. The interface device used to alter the voltage, delivered by the respiratory belt transducer, is shown in

caudal-cranial direction $[7-9,11,12]$. Since a sub-centimeter precision is often required for effective therapy, a technique to overcome interference of RICM during ESM procedures is desirable.

Fusion of ESM data with surface meshes obtained from pre-procedurally acquired 3D imaging data from, e.g., CMR or SPECT/CT has recently gained more interest in order to optimize catheter interventions $[3,5,10,11,13$, 14]. Since registration of the imaging modalities is done using landmark or surface registration, minimization of the spatial error is of great importance. RICM artifacts can lead to a misalignment in the registration process; therefore, compensation for RICM without the use of respiratory gating [1] will reduce procedure time and improve registration accuracy.

In this study, we introduce a novel technique to correct for the effect of RICM during ESM procedures without the need for respiratory gating. The NOGA ${ }^{\circledR} \mathrm{XP}$ (Biosense Webster, Cordis, Johnson \& Johnson, version 1.1.43) intramyocardial injection system was used which provides a 3D magnetic tracking of the catheter and measurement of local electrical and mechanical tissue characteristics. Since the NOGA ${ }^{\circledR} \mathrm{XP}$ system is not able to measure respiration, a simple add-on was developed to enable respiratory registration through available electrocardiogram (ECG) leads.

We identified the effect of RICM on the ESM accuracy during mapping procedures by comparing ESM data with gold standard CMR-derived surface meshes in a porcine study. The goal was twofold: (1) to minimize RICM artifacts in constructed ESM and (2) to decrease the registration error in the fusion of ESMs with CMR. the center panel. The interface device is connected to the left leg (LL) and V1 ECG channels on the NOGA ${ }^{\circledR}$ XP PUI box. The respiratory belt transducer is connected to the interface device using a regular BNC connector

\section{Methods}

\subsection{Respiration and NOGA measurements}

A piezo-respiratory belt transducer (UFI Model 1132 Pneumotrace $\mathrm{II}^{\mathrm{TM}}$ ) was connected to an ECG input of the $\mathrm{NOGA}^{\circledR} \mathrm{XP}$ system (Fig. 1). The respiratory signal was represented on the $\mathrm{NOGA}^{\circledR} \mathrm{XP}$ system as a baseline shift of the connected ECG channel. Prior to the experiment, the potentiometer in the device was adjusted so that the connected ECG channel showed a clear baseline drift. The NOGA ${ }^{\circledR} \mathrm{XP}$ system stores end-diastolic 3D location of each acquired location in the local database using the built-in ECG $R$-wave triggering. The NOGA ${ }^{\circledR} \mathrm{XP}$ system records a 12-lead ECG with a length of $2.5 \mathrm{~s}$ per measured point. The sample rate of the signal is $1 \mathrm{kHz}$. The NOGA ${ }^{\circledR} \mathrm{XP}$ ECG filter settings were optimized to allow visualization of respiratory signals in the $0.05-0.5 \mathrm{~Hz}$ range. An external computer was used to access the ECG data on the NOGA ${ }^{\circledR} \mathrm{XP}$ system via a shared folder. The NOGA ${ }^{\circledR} \mathrm{XP}$ database was accessed through a SQL server with read-only permissions.

\subsection{Phantom experiment}

A non-rotational symmetric bottle was mapped from the inside in a stationary position. Thereafter, the phantom was mounted on a test lung that was connected to a ventilator. The ventilator tidal volume was set to induce a $15-\mathrm{mm}$ motion of the phantom in primarily one direction. To acquire a reference measurement, the 7 French NOGA $^{\circledR} \mathrm{XP}$ mapping catheter (Biosense Webster, Cordis, Johnson \& Johnson, Diamond Bar, USA) was fixed to the phantom 
while ventilating the test lung. Hereafter, the phantom was mapped on the inside while ventilating the test lung. During the mapping procedures, an ECG simulator was connected to the $\mathrm{NOGA}^{\circledR} \mathrm{XP}$ system to enable triggered data acquisition. The ECG simulator was set to simulate normal sinus rhythm at 80 beats/min.

\subsection{Porcine experiments}

All animal experiments were conducted in accordance with the national guidelines on animal care and with prior approval by the Animal Experimentation Committee of the Faculty of Medicine, Utrecht University, the Netherlands. Five female Dallas Landrace pigs were subjected to a 90-min left anterior descending balloon occlusion and subsequent reperfusion 8 weeks before the measurements.

A respiratory belt transducer was attached around the abdomen in the sub-thoracic region, where maximal respiratory-induced abdominal movement could be measured. The transducer was connected to the ESM system as described before. The ventilator was set to a frequency of 12 breaths per minute with a tidal volume of $800 \mathrm{ml}$ and a 2:1 expiration to inspiration ratio. The $\mathrm{NOGA}^{\circledR} \mathrm{XP}$ catheter was inserted into the left ventricle (LV) via the left carotid artery and retrograde passage through the aortic valve. First, the catheter was positioned in the LV apex and a reference measurement was taken containing at least 60 measurements to assure measurements in all phases of the respiratory cycle. Secondly, in an independent procedure, a complete map of the LV was made. After the mapping procedures, a CMR scan was made. The animals were euthanized after finishing the experiments.

\subsection{CMR}

In vivo CMR was performed under anesthesia on a clinical 3T scanner (Achieva TX, Philips Healthcare, Best, the Netherlands) with a 32-channel receiver coil.

ECG-gated cine images were made with voxel size $=2 \times 2 \mathrm{~mm}$, flip angle $=45^{\circ}$, slice thickness $=8 \mathrm{~mm}$, field of view $=320 \times 320 \mathrm{~mm}$, repetition time $[\mathrm{TR}]=3.2 \mathrm{~ms}$, echo time $[\mathrm{TE}]=1.6 \mathrm{~ms}$ and 30 phases/R-to-R interval.

End-diastolic frames of the cine images were segmented using the freely available software Segment version 1.9 R3262 (http://segment.heiberg.se) [6]. An end-diastolic endocardial surface mesh was generated and used for the registration of the $\mathrm{NOGA}^{\circledR} \mathrm{XP}$ mapping points. The mesh creation and registration were performed using the in-house developed 3D CartBox and were based on a standard rotation and translation followed by an angle-limited iterative closest point procedure and manually corrected if necessary [14].

\subsection{Correction algorithm}

A detailed overview of the correction algorithm is shown in Fig. 2. As a first step, a reference measurement was taken to assess the RICM. In the phantom experiment, the catheter tip was mounted at a fixed surface location during movement of the phantom. In the in vivo experiments, the catheter was positioned in the LV apex during the reference measurements. In both experiments, at least 60 measurements were used for the reference measurement. It was assured that points and measurements were located in every phase of the respiratory cycle. Storage of the 3D location of the catheter tip is triggered at $t=2 \mathrm{~s}$ in the stored ECG. For the respiratory analysis, the ECG signal containing the respiratory data was fitted with a fifth-order polynomial function over the 1.5 - to 2.5 -s interval to eliminate $R$-wave artifacts and signal noise (MATLAB 2013a version 8.1, The MathWorks Inc.). The data were split into an inspiratory and expiratory part based on the slope $(\theta)$ of the respiratory signal at $t=2 \mathrm{~s}$ (Fig. 2a). For both parts, the position is defined in the $X, Y$ and $Z$ directions. For each direction, a second-order polynomial function is fitted using a least squares approach (Eq. 1) (e.g., Fig. 3b, e). The six resulting functions were thereafter used during the mapping procedure to calculate the correction of every acquired point based on the value $(\xi)$ and $\theta$ of the respiratory signal (Eq. 2).

$f_{c}=a x^{2}+b x+c$

The second-order polynomial function that was used to fit the data acquired during the reference measurement. This was done for each direction for both the inspiratory and expiratory data. $f_{\mathrm{c}}$ represents the measured values, and parameters $a, b$ and $c$ are determined and used to subsequently calculate the correction during the mapping procedure.

$\left[\begin{array}{l}x_{\mathrm{c}} \\ y_{\mathrm{c}} \\ z_{\mathrm{c}}\end{array}\right]=\left[\begin{array}{l}x_{\mathrm{m}} \\ y_{\mathrm{m}} \\ z_{\mathrm{m}}\end{array}\right]-\left[\begin{array}{l}f_{\mathrm{x}}(\xi) \\ f_{\mathrm{y}}(\xi) \\ f_{\mathrm{z}}(\xi)\end{array}\right]$

The equation that was used to correct the acquired mapping data, $f_{x}(\xi)$, represents the polynomial function best fitting the $X$-coordinate data from the reference measurement shown in Eq. 1, and $\xi$ represents the $y$ value measured in the normalized respiratory signal. The corrected and measured $X$-coordinate is represented by $X_{\mathrm{c}}$ and $X_{\mathrm{m}}$, respectively.

\subsection{Analysis and statistics}

The algorithm is used to relocate measurement points to their location at maximal expiration. To assess the effects of the RICM correction, the root-mean-squared error (RMSE) of the geometrical distance from all points of the reference 


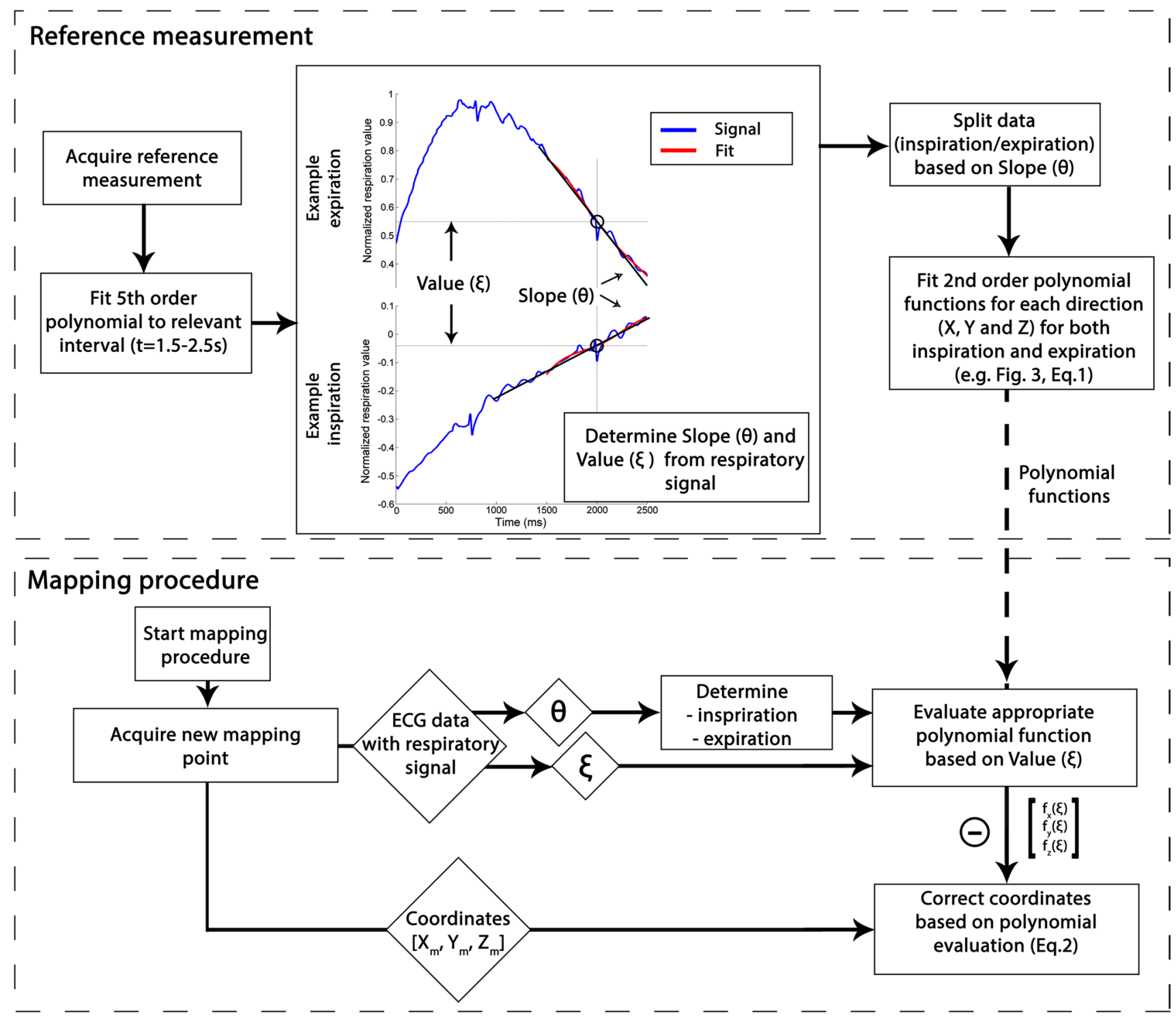

Fig. 2 The upper panel shows the flowchart of the algorithm that was used to determine the correction parameters from the reference measurement. The bottom how these parameters were used to correct

measurement to the point acquired at maximal expiration was calculated. The same end-expiratory location was used for both the uncorrected and corrected RMSE. Maximal apical displacement represents the maximal range of all apical locations during the reference measurement, i.e., the distance between the point at maximal expiration and the point furthest from that. Both RMSE and the maximal apical displacement are expressed in millimeters. The registration error is calculated as mean distance of each mapping point to the CMR end-diastolic endocardial surface mesh after registration of the NOGA ${ }^{\circledR} \mathrm{XP}$ ESM with the endocardial surface mesh generated from cine CMR. The registration error is expressed as mean \pm standard deviation acquired point during the mapping procedure. $\theta=$ slope, $\xi=y$ value, $X_{\mathrm{m}}=$ measured coordinate

in millimeters. Paired $t$ tests were used to compare original with corrected data, and $p<0.05$ was considered to be statistically significant.

\section{Results}

\subsection{Respiratory registration}

Recording respiratory signals on a precordial ECG lead were performed successfully. The respiratory-induced baseline shift in ECG lead V1 during the phantom and in vivo experiments is shown in Fig. 3a, d, respectively. 

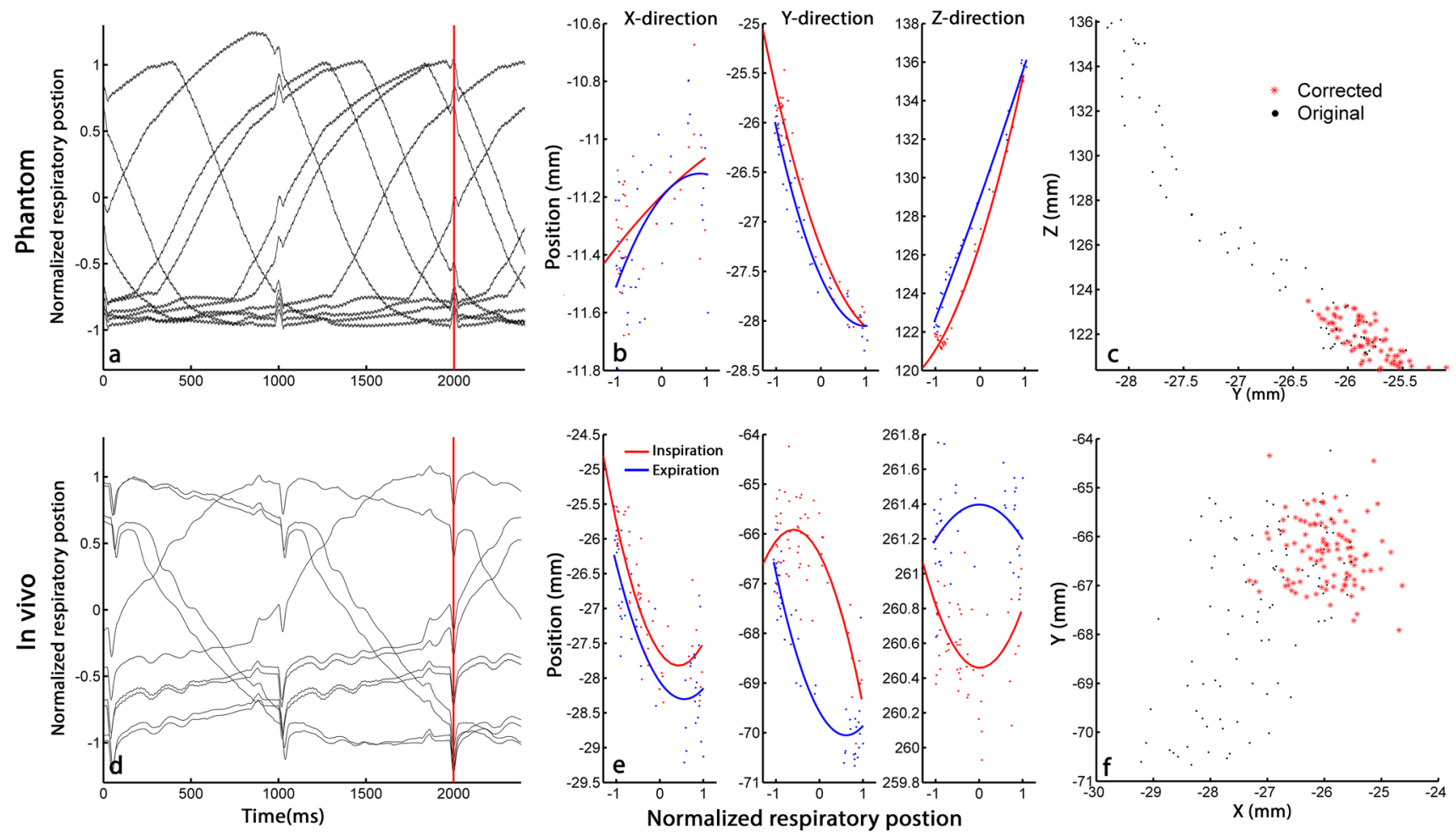

Fig. 3 a, d Example of the first ten registered respiratory signals recorded on the precordial ECG V1 lead in the phantom and in vivo experiments, respectively. The respiratory position is determined at time point $t=2 \mathrm{~s}$ (red line), the end-diastolic phase ( $R$-wave). b, e The recorded 3-dimensional $(X, Y$ and $Z)$ data of all $(n=77$ and $n=100$, respectively) mapped reference points with the fitted sec-

\subsection{Phantom experiment}

In the motionless phantom, 142 positions were mapped; in the moving phantom, 70 and 118 points were, respectively, acquired during the reference and mapping measurements. In the reference measurement, the derived motion of the phantom was $15 \mathrm{~mm}$ in the $Z$ direction and $2 \mathrm{~mm}$ in the $X$ and $Y$ directions (Fig. 3b). The result of the correction algorithm on the motion of the reference measurement is shown in Fig. 3c. The registration error of the ESM on the CMR was $2.0 \pm 1.4 \mathrm{~mm}$ when no respiratory motion was applied to the phantom (Fig. 4a). With motion, the registration error was $2.8 \pm 2.2 \mathrm{~mm}$ before and $1.9 \pm 1.3 \mathrm{~mm}$ after correcting for the motion (Figs. $4 \mathrm{~b}, \mathrm{c}$ and $5 \mathrm{~b}$; Table 1). The RMSE in the reference measurement was $6.5 \mathrm{~mm}$ before and $1.1 \mathrm{~mm}$ after correction (Fig. 5a).

\subsection{Porcine experiments}

In five animals ( $83 \pm 6 \mathrm{~kg}$ ), on average, 91 (range 75-101) reference points were acquired in the apex and the mean number of points in the left ventricular maps was 80 (range 63-90). In four animals, the baseline shift in the ond-order polynomial functions for inspiration (red) and expiration (blue). Note the differences in scale on the vertical axis. c, f Reference measurement before (black) and after correction (red). This is a 2-dimensional representation of the 3D data in the two directions that show the largest variation

respiratory signal was successfully recorded. The RICM correction algorithm was able to correct for the respiratoryinduced apical movement (Fig. 5a; Table 1). The average apical displacement (RMSE) was reduced by $51 \%$ from $2.6 \pm 1.0 \mathrm{~mm}$ before to $1.2 \pm 0.3 \mathrm{~mm}$ after correction $(p<0.05)$. The maximal apical displacement was reduced by $34 \%$ from $3.7 \pm 1.1 \mathrm{~mm}$ before to $2.3 \pm 0.5 \mathrm{~mm}$ after correction $(p=0.09)$. A representative example of acquired apical reference points is shown in Fig. 3f. The registration error was not significantly $(p=0.62)$ reduced from $3.8 \pm 0.5$ to $3.7 \pm 0.4 \mathrm{~mm}$ (mean $\pm \mathrm{SEM}$ ) after correction.

\section{Discussion}

Measuring RICM with the NOGA ${ }^{\circledR} \mathrm{XP}$ system is feasible using our in-house developed customized interface. In a phantom experiment, the correction algorithm showed to be effective in reducing the RMSE of the reference measurement and the registration error of the $\mathrm{NOGA}^{\circledR} \mathrm{XP}$ ESM and the CMR dataset. In the porcine experiments, RICM of the LV apex was smaller than values reported in human studies. Our correction algorithm is, however, capable 

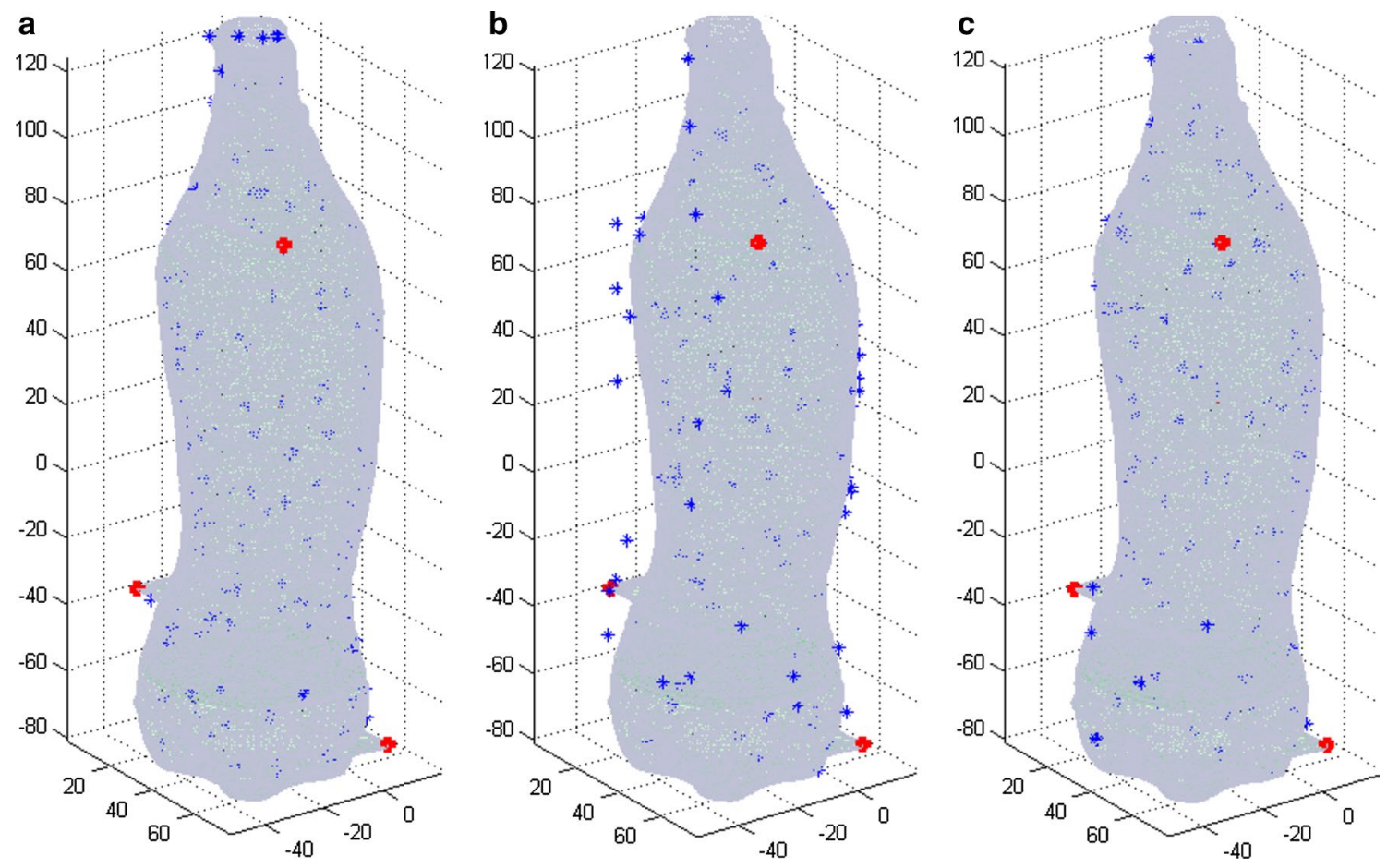

Fig. 4 a The registration of acquired ESM points (blue) to the MRIderived surface mesh (light blue) where no motion was applied to the phantom. Landmark points (red) were fixed to the external surface of the phantom (registration error $2.0 \pm 1.4 \mathrm{~mm}$ ). b The uncorrected registration with applied motion (registration error $2.8 \pm 2.2 \mathrm{~mm}$ ). c The corrected registration with applied motion (registration error $1.9 \pm 1.3 \mathrm{~mm})$
Fig. 5 a The RMSE error of the reference measurement before and after correction. $\mathbf{b}$ The registration error of the ESM map and the MRI-derived endocardial surface mesh before and after correction. The phantom where no motion was applied (Ph. still) was not corrected. $P h$. phantom
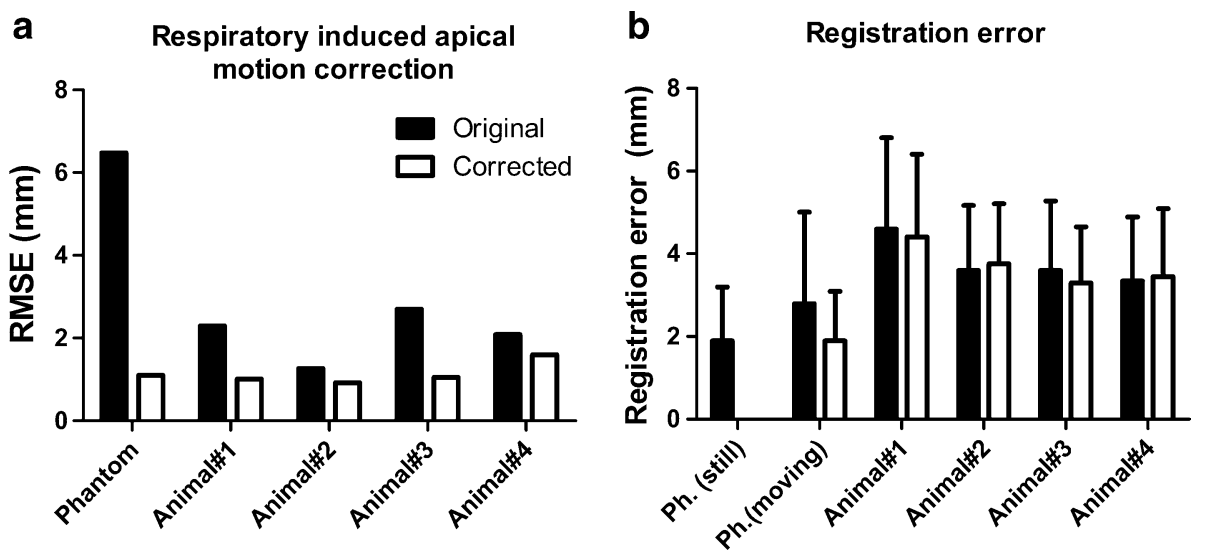

Table 1 Individual results of all experiments

\begin{tabular}{|c|c|c|c|c|c|c|}
\hline & \multicolumn{2}{|c|}{ Apex movement RMSE (mm) } & \multicolumn{2}{|c|}{$\begin{array}{l}\text { Apex maximal displacement } \\
(\mathrm{mm})\end{array}$} & \multicolumn{2}{|c|}{ Registration error (mm) } \\
\hline & Original & Corrected & Original & Corrected & Original & Corrected \\
\hline Phantom & 6.5 & 1.1 & 14.0 & 2.02 & $2.8 \pm 2.2$ & $1.9 \pm 1.2$ \\
\hline Animal\#1 & 2.3 & 1.0 & 3.4 & 1.95 & $4.6 \pm 2.2$ & $4.4 \pm 2.0$ \\
\hline Animal\#2 & 1.3 & 0.9 & 2.6 & 1.67 & $3.6 \pm 1.6$ & $3.8 \pm 1.5$ \\
\hline Animal\#3 & 2.7 & 1.6 & 5.5 & 2.62 & $3.6 \pm 1.7$ & $3.3 \pm 1.4$ \\
\hline Animal\#4 & 4.1 & 1.7 & 3.3 & 3.06 & $3.3 \pm 1.6$ & $3.4 \pm 1.7$ \\
\hline
\end{tabular}


of significantly reducing (34\%) the apical displacement throughout the respiratory cycle. The reduction in registration error of corrected ESM data on the CMR data was minimal. This most likely is due to the minimal amount of RICM observed in this porcine model. One animal was excluded from analysis due to a malfunctioning respiration transducer.

Multiple studies into RICM report movement of the human heart in the range of one centimeter [7-9, 11, 12]. In the present study, the maximal apical displacement measured was $3.7 \pm 1.1 \mathrm{~mm}$. An explanation for this difference might be found in the difference of thoracic anatomy between humans and pigs. The thoracic anatomy of the pig might lead to reduced RICM. Furthermore, to explore the RICM of pigs, we set up a single experiment in which we increased the tidal volume. We observed a $120 \%$ increase (3.3-7.3 mm) in RICM (maximal apical displacement) of the apex upon a $20 \%$ increase $(800-950 \mathrm{ml})$ in tidal volume. Although a tidal volume of $800 \mathrm{ml}$ was used during the experiments, the design and validation of the RICM algorithm can be done accurately with the used setup.

Multiple commercially available ESM systems offer the option to use respiratory gating in addition to ECG gating during ESM procedures. This inevitably leads to longer procedure times since mapping points are only acquired during a short phase of the respiratory cycle, usually the end-expiratory phase [1]. Furthermore, respiratory gating in free-breathing patients may be unreliable since respiratory cycles are never identical. This leads to different residual volumes and thus to different positions of the heart during end expiration. Our respiratory correction algorithm allows the acquisition of mapping points during the whole respiratory cycle. By uncoupling inspiration and expiration, and taking both the slope $(\theta)$ and value $(\xi)$ into account during the analysis, the algorithm is able to minimize problems associated with breath to breath variability in tidal volume. Since the algorithm depends on reference measurements, subject-specific breathing characteristics, such as depth and rate of breathing, are considered in the algorithm. Additionally, using these characteristics, abnormal breaths during the mapping procedure can be identified automatically and excluded if necessary.

\subsection{Data acquisition}

Acquiring respiration data with the $\mathrm{NOGA}^{\circledR} \mathrm{XP}$ system using ECG input ports are feasible since our customized device uses only one of the precordial leads (e.g., V1), which are often unused during mapping/injection procedures. The use of an ECG lead as an input for the respiratory signal ensures synchronicity of the stored signals and location data. For use in a clinical setting, a dedicated solution is desirable to allow different settings of, for example, the filter settings. In this study, no difficulties in visualizing neither the ECG signals nor the respiratory signals were found.

\subsection{Limitations}

All experiments were performed with the animal connected to a ventilator; therefore, respiration curves were predictable and similar throughout the entire procedure. RICM in the porcine model is minimal, and as a result, it is difficult to predict the effect of RICM on the registration error in a clinical situation.

\subsection{Clinical perspective}

Many imaging modalities, such as late gadolinium enhancement CMR, provide detailed information about the infarct location and its characteristics. Combining or fusing ESM data with, for example, late gadolinium enhancement CMR may improve therapeutic outcome $[10,13,16]$. The registration error between ESMs and, for example, MRI data is typically around $3 \mathrm{~mm}$ in animals and humans (e.g., $3.22 \pm 1.86 \mathrm{~mm}[14]$ and $3.8 \pm 0.6 \mathrm{~mm}$ [15], respectively). Since RICM in patients is often much larger [7-9, 11, 12], correction of RICM will probably lead to fewer registration artifacts and therefore to more accurate multimodality catheter diagnosis and treatment. The validation of the correction algorithm in this study was performed retrospectively. However, the correction algorithm is intended to be used in real time during ESM mapping procedures. For example, the reference measurement takes about 1-2 min, and calculating parameters needed for the subsequent procedure requires less than $2 \mathrm{~s}$ on a modern computer. During the actual mapping procedure, calculating the correction for a single acquired point requires a few milliseconds. Use of the correction algorithm in real time will therefore not affect the length of the ESM procedure.

\section{Conclusion}

The correction of RICM during ESM procedures by our correction algorithm is feasible and can be incorporated in commercially available systems. In contrast to the phantom experiments, the suggested RICM correction method was of less additional value during porcine experiments due to minimal RICM in the porcine model. Nevertheless, during patient procedures, RICM correction can be of additional value to obtain an anatomically correct map and allows a more optimal registration with respiratorygated cardiac imaging data. A new clinical study must be performed to investigate the clinical benefit of RICM correction. 
Acknowledgments We kindly acknowledge Grace Croft, Marlijn Jansen, Evelyn Velema, Joyce Visser, Merel Schuurink, Martijn van Nieuwburg and Gerard Marchal for their assistance with the animal experiments. We would like to thank Joep van Oorschot, Jaco Zwanenburg and Martijn Froeling for performing the CMR experiments.

\section{Compliance with ethical standards}

Conflict of interest This research forms part of the Project P1.04 SMARTCARE of the research program of the BioMedical Materials Institute, co-funded by the Dutch Ministry of Economic Affairs, Agriculture and Innovation. The financial contribution of the Nederlandse Hartstichting (NHS) is gratefully acknowledged. Author F.J. van Slochteren is co-founder of CART-Tech B.V.

Ethical approval All applicable international, national and/or institutional guidelines for the care and use of animals were followed.

Open Access This article is distributed under the terms of the Creative Commons Attribution 4.0 International License (http://creativecommons.org/licenses/by/4.0/), which permits unrestricted use, distribution, and reproduction in any medium, provided you give appropriate credit to the original author(s) and the source, provide a link to the Creative Commons license, and indicate if changes were made.

\section{References}

1. Beinart R, Kabra R, Heist KE et al (2011) Respiratory compensation improves the accuracy of electroanatomic mapping of the left atrium and pulmonary veins during atrial fibrillation ablation. J Interv Cardiac Electrophysiol Int J Arrhythmias Pacing 32:105-110. doi:10.1007/s10840-011-9583-z

2. Ben-Haim SA, Osadchy D, Schuster I, Gepstein L, Hayam G, Josephson ME (1996) Nonfluoroscopic, in vivo navigation and mapping technology. Nat Med 2:1393-1395

3. Dauwe DF, Nuyens D, De Buck S et al (2014) Three-dimensional rotational angiography fused with multimodal imaging modalities for targeted endomyocardial injections in the ischaemic heart. Eur Heart J Cardiovasc Imaging 15:900-907. doi:10.1093/ehjci/jeu019

4. De Groot NM, Bootsma M, van der Velde ET, Schalij MJ (2000) Three-dimensional catheter positioning during radiofrequency ablation in patients: first application of a real-time position management system. J Cardiovasc Electrophysiol 11:1183-1192

5. Dickfeld T, Tian J, Ahmad G et al (2011) MRI-Guided ventricular tachycardia ablation: integration of late gadolinium-enhanced 3D scar in patients with implantable cardioverter-defibrillators. Circ Arrhythmia Electrophysiol 4:172-184. doi:10.1161/ CIRCEP.110.958744

6. Heiberg E, Sjögren J, Ugander M et al (2010) Design and validation of segment-freely available software for cardiovascular image analysis. BMC Med Imaging 10:1. doi:10.1186/1471-2342-10-1

7. Holland E, Goldfarb W, Edelman R (1998) Diaphragmatic and cardiac motion during suspended breathing: preliminary experience and implications for breath-hold. Radiology 209:483-489

8. Jagsi R, Moran JM, Kessler ML et al (2007) Respiratory motion of the heart and positional reproducibility under active breathing control. Int J Radiat Oncol Biol Phys 68:253-258. doi:10.1016/j. ijrobp.2006.12.058

9. Qi XS, White J, Rabinovitch R et al (2010) Respiratory organ motion and dosimetric impact on breast and nodal irradiation. Int J Radiat Oncol Biol Phys 78:609-617. doi:10.1016/j. ijrobp.2009.11.053

10. Reddy VY, Malchano ZJ, Holmvang G et al (2004) Integration of cardiac magnetic resonance imaging with three-dimensional electroanatomic mapping to guide left ventricular catheter manipulation feasibility in a porcine model of healed myocardial infarction. J Am College Cardiol. doi:10.1016/j.jacc.2004.08.063

11. Roujol S, Anter E, Josephson ME, Nezafat R (2013) Characterization of respiratory and cardiac motion from electro-anatomical mapping data for improved fusion of MRI to left ventricular electrograms. PLoS One 8:e78852. doi:10.1371/journal. pone. 0078852

12. Shechter G, Ozturk C, Resar JR, Mcveigh ER (2004) Respiratory motion of the heart from free breathing coronary angiograms. IEEE Trans Med Imaging 23:1046-1056. doi:10.1109/ TMI.2004.828676

13. Tao Q, Milles J et al (2011) Toward magnetic resonanceguided electroanatomical voltage mapping for catheter ablation of scar-related ventricular tachycardia: a comparison of registration methods. J Cardiovasc Electrophysiol. doi:10.1111/j.1540-8167.2011.02167.x

14. van Slochteren FJ, van Es R, Koudstaal S et al (2014) Multimodality infarct identification for optimal image-guided intramyocardial cell injections. Neth Heart J 22:493-500. doi:10.1007/ s12471-014-0604-2

15. Wijnmaalen AP, Van Der Geest RJ, Van Huls Van Taxis CFB et al (2011) Head-to-head comparison of contrast-enhanced magnetic resonance imaging and electroanatomical voltage mapping to assess post-infarct scar characteristics in patients with ventricular tachycardias: real-time image integration and reversed registration. Eur Heart J 32:104-114. doi:10.1093/eurheartj/ehq345

16. Wittkampf FH, Wever EF, Derksen R et al (1999) LocaLisa: new technique for real-time 3-dimensional localization of regular intracardiac electrodes. Circulation 99:1312-1317. doi:10.1161/01.CIR.99.10.1312

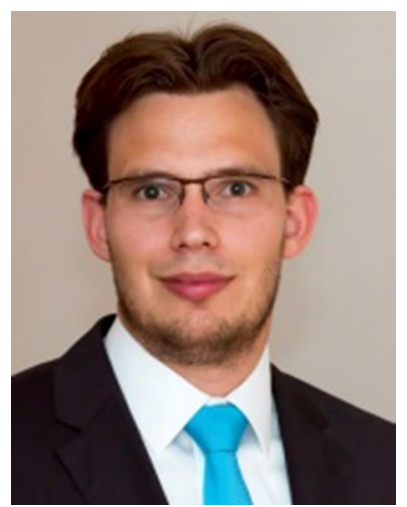

René van Es M.Sc. was trained as a technical physician. His research focuses on the improvement of catheter-based therapies. René is currently working as a Ph.D. student in the Department of Cardiology. 


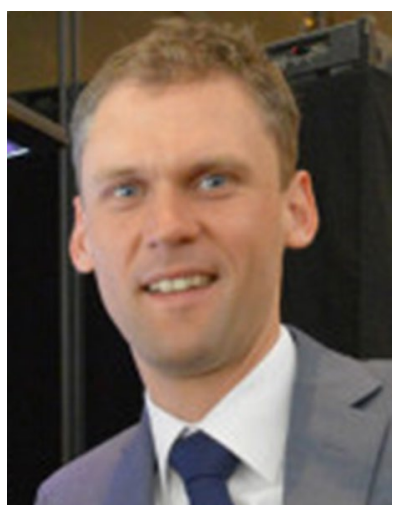

Frebus J. van Slochteren

Ph.D. is trained as a medical engineer. He obtained his Ph.D. degree from the University of Utrecht in 2014. His Ph.D. thesis was titled: 'Technical solutions to improve cardiac regenerative therapy.' $\mathrm{He}$ is the co-founder of CART-Tech B.V.

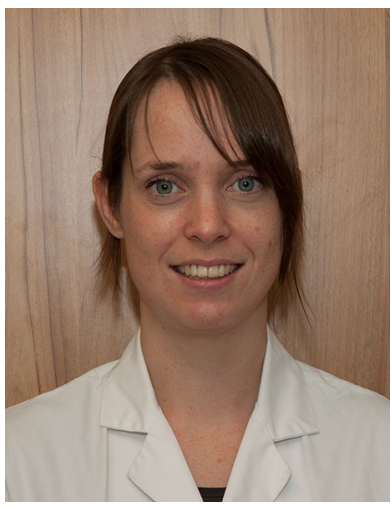

Sanne J. Jansen of Lorkeers MD Ph.D. researched on cardiac regeneration, but also on transparency and methodology of preclinical research in order to improve clinical translation. She is currently working as a cardiology resident.

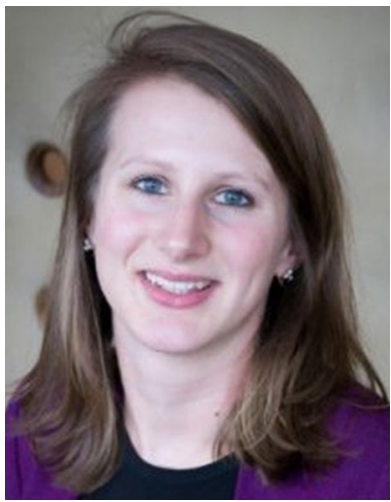

Roos Blankena M.Sc. was trained as technical physician and did, next to this study, research in MR imaging of cerebral aneurysm walls. She is currently working as consultant health care at IG\&H Consulting \& Interim.

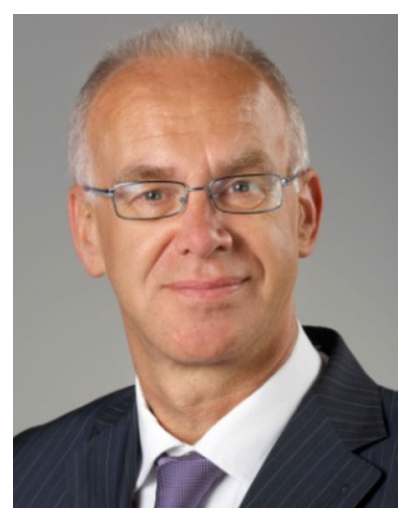

Pieter A. Doevendans MD

Ph.D. was trained as an interventional cardiologist and was appointed full professor in Translational Cardiology. He is currently chairman and medical manager of Division Heart and Lungs, in the University Medical Center Utrecht.

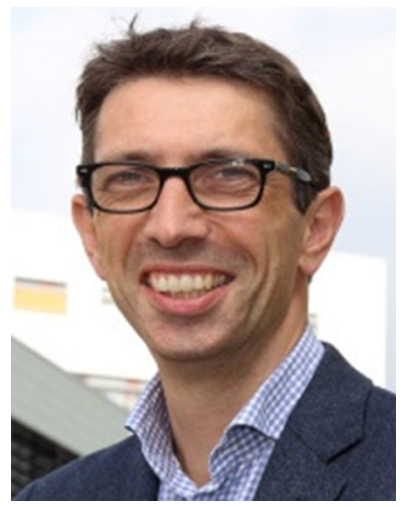

Steven A.J. Chamuleau MD Ph.D. is a cardiologist and associate professor at the University Medical Center Utrecht specialized in cardiac regenerative therapy and cardiac imaging. He combines state-of-theart care with promising experimental techniques. 